\title{
QoE-Centric Management of Multimedia Networks through Cooperative Control Loops
}

\author{
Niels Bouten, Steven Latré, and Filip De Turck \\ Department of Information Technology, Ghent University - iMinds \\ \{niels.bouten, steven.latre,filip.deturck\}@intec. ugent.be \\ http://ibcn.intec.ugent.be
}

\begin{abstract}
The Internet has evolved from a service to transport simple text files into a platform for transporting a variety of complex multimedia services. The initial centralized management systems were not designed and are therefore not able to perform efficient management of Quality of Experience (QoE) for these complex services. Deploying an autonomic management system resolves these complexity issues and allows efficient resource allocation based on the service type, end-user requirements and device characteristics. However, existing autonomic management systems only allow limited cooperation between different autonomic elements (AE), which limits their capabilities to provide endto-end QoE assurance. This research will therefore design cooperative AEs, optimize their organization and provide cooperative allocation algorithms to optimize end-to-end QoE.
\end{abstract}

Keywords: Autonomic Network Management, Multimedia Services.

\section{Introduction}

The consumption of multimedia services over the Internet has increased tremendously over the past decade and this growth is forecasted to continue over the next years [1. Not only the variety of services has expanded, also the end-user devices used to access those services have diversified. These heterogeneous services and devices have more stringent demands in terms of bitrate, maximum packet loss and maximum latency, than the services for which the Internet was initially designed. To be able to cope with this increased demands, providers tend to over-dimension the available resources to be able to meet the Quality of Service (QoS) levels at all times. These are however expensive upgrades for which end-users pay for in the end. The problems however are not caused by a lack of infrastructure, but are induced by an inefficient allocation of the available resources. The overall quality as perceived by the end user needs to be expressed in terms of Quality of Experience (QoE) and can not be achieved by assigning equal bitrates to each data flow 2. Although QoE is related to QoS, there is no direct mapping between both concepts: a small percentage of packet loss will yield acceptable QoE for a data transmission service, but the same QoS-levels will yield artifacts when applied to a video streaming, deteriorating QoE. The allocation of resources to data flows therefore needs to include flow characteristics 
(e.g., the content of the flow, user subscription level, end-user device properties) during the decision process.

These requirements induce a steep increase in complexity for the centralized network management software, leading to complicated systems for the operators and towering maintenance costs. Therefore, the autonomic networking paradigm proposes to shift the workload to the system itself by using distributed autonomic elements (AEs) which are responsible for configuring the system and react to novel situations without requiring time-consuming manual interventions by human operators. Existing autonomic systems however only allow limited cooperation between the individual control loops, potentially leading to counteracting decisions. To be able to provide end-to-end quality assurance, the autonomic control loops need to cooperate in order to provide an optimal solution.

\section{QoE-Centric Management}

To be able to cope with the increasing complexity of network management software, a network of distributed AEs needs to be deployed. These AEs each manage one or more QoE-optimizers (e.g., rate adaptation system, access control system, caching infrastructure). Existing autonomic systems use isolated elements which take their decisions independently from other elements. Figure 1 shows how the lack of cooperation between elements prevents the management system to provide optimal end-to-end quality assurance. When congestion arises on the delivery path between the end-users and the video source (1), this will be signaled to both AEs along this path (2). Since the AEs do not cooperate, both of them will decide to take action: sessions will be declined (3) and the video bitrate will be lowered to eliminate congestion (4). In this way, less users will be admitted and they will receive a degraded version of the video, deteriorating overall QoE due to the lack of cooperation.

Enabling cooperation between AEs does not only require extension of existing control loops such as the FOCALE control loops [3] to support communication

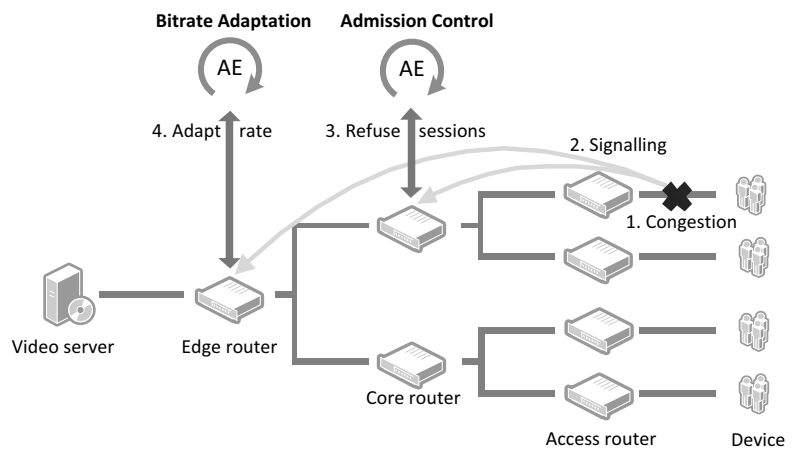

Fig. 1. Example of conflicting decisions between AEs when no cooperation is present, leading to inefficient use of resources and a drop of QoE 
and knowledge exchange, they also need to be structured to form a scalable and reliable network management overlay. The impact of a registry-based, multiagent [4] and hierarchical organization [5] of AEs in terms of scalability, efficiency, reliability and convergence needs to be quantified. One of the drawbacks of a registry-based organization is the use of a centralized repository, which undermines the scalability and reliability of the system. Multi-agent based organization allows flexible interaction between groups of agents via a shared bus. Although providing solutions for scalability and reliability, the lack of global information can lead to divergent decisions between AEs. A hierarchical approach overcomes these issues, since the root has access to aggregate information on the entire system, but at the same time this introduces trust issues when orchestrating cooperation beyond the borders of management domains since control needs to be transferred to a third party entity. Taking into account the previous remarks, a hybrid solution proposes itself where resources are managed hierarchically within a certain domain with a multi-agent based organization across management domains.

In order to provide end-to-end quality management, distributed decision algorithms need to be deployed in the autonomic management overlay. The goal of these algorithms is to keep track of the available resources, identify which actions are required in the network and which components are able to complete these actions. A first key step to attain QoE-centric management is to identify the impact of the managed components on QoE and model the different states, actions and transitions using finite state machines. This allows the autonomic system to analyze the network condition, determine the corresponding state and identify the possible QoE-improving actions that are available. A second step is to cooperatively decide which QoE-optimizers will be deployed and whom should manage them. The third step in achieving autonomic QoE-centric management is to dynamically adapt the constructed state models to be able to cope with unknown situations.

\section{Obtained Results}

During the first year of the $\mathrm{PhD}$, the scalability issues arising from replicated unicast HTTP Adaptive Streaming (HAS) sessions were addressed 6]. A hierarchical approach was taken, where the autonomic distribution management collects session data from the delivery servers, analyzes this and decides which sessions will be grouped. The autonomic delivery management then decides which multicast channels it wants to receive. This approach led to a considerable decrease in bandwidth consumption (i.e. $23 \%$ when applying autonomic distribution management and an additional 25\% when applying autonomic channel selection at the delivery server) while increasing the QoE. The disadvantages of the absence of cooperation were also discussed in previous work, showing the difficulty of competing autonomic HAS clients to converge to a stable solution 7]. By deploying additional in-network management, taking into account the subscription and device parameters of the clients, the stability and performance of autonomic HAS clients was improved, leading to a higher overall QoE. 


\section{Conclusion}

To overcome QoE-management issues in centralized network management systems, this paper proposes to apply autonomic management for end-to-end QoE optimization. This is obtained using an overlay of cooperative AEs which prevents contradictory decisions, deteriorating end-to-end QoE. Using a hybrid approach between a hierarchical and agent-based organization overcomes issues with a trusted third party entity and lack of global information that arise in respectively hierarchical and multi-agent organization. In order to support endto-end QoE, distributed allocation algorithms need to be deployed to manage the available resources efficiently. Initial results show the benefits of applying an autonomic management overlay to HTTP Adaptive Streaming services. In future work, the impact of the organization of AEs on efficiency and reliability will be investigated together with how inter-domain cooperation is affected by the organization scheme.

Acknowledgements. Niels Bouten is funded by a Ph.D. grant of the Agency for Innovation by Science and Technology (IWT). This work was partly funded by Flamingo, a Network of Excellence project (ICT-318488) supported by the European Commission under its Seventh Framework Programme.

\section{References}

1. CISCO. Cisco Visual Networking Index: 2011-2016. Technical Report (2012)

2. Briscoe, B.: Flow rate fairness: Dismantling a religion. ACM SIGCOMM Computer Communications Review 37(2), 63-74 (2007)

3. Agoulmine, N.: Autonomic Network Management Principles: From Concepts to Applications. Academic Press - Elsevier (2011)

4. Tesaureo, G., Chess, D.M., Walsh, W.E., Das, R., Segal, A., Whalley, I., Kephart, J.O., White, S.R.: A Multi-Agent Systems Approach to Autonomic Computing. In: Proceedings of the Third International Joint Conference on Autonomous Agents and Multiagent Systems, vol. 1, pp. 464-471. IEEE Computer Society (2004)

5. Famaey, J., Latré, S., Strassner, J.: A Hierarchical Approach to Autonomic Network Management. In: Proceedings of the 2nd IFIP/IEEE International Workshop on Management of the Future Internet (ManFI), pp. 225-232 (2010)

6. Bouten, N., Latré, S., Van De Meerssche, W., De Vleeschauwer, B., De Schepper, K., Van Leekwijck, W., De Turck, F.: A multicast-enabled delivery framework for QoE assurance of over-the-top services in multimedia access networks. Journal of Network and Systems Management, 1-30 (2013)

7. Bouten, N., Famaey, J., Latré, S., Huysegems, R., De Vleeschauwer, B., Van Leekwijck, W., De Turck, F.: QoE Optimization Through In-Network Quality Adaptation for HTTP Adaptive Streaming. In: 8th International Conference on Network and Service Management (CNSM), pp. 336-342. IEEE (2012) 